\title{
Pressure Generation from Micro-Bubble Collapse at Shock Wave Loading*
}

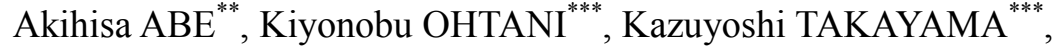 \\ Shigeru NISHIO $^{* *}$, Haruo MIMURA ${ }^{* *}$ and Minoru TAKEDA ${ }^{* *}$ \\ ${ }^{* *}$ Graduate School of Maritime Sciences, Kobe University, \\ 5-1-1 Fukaeminami-machi, Higashinada-ku, Kobe 658-0022, Hyogo, Japan \\ E-mail: a-abe@maritime.kobe-u.ac.jp \\ ${ }^{* * *}$ Shock Wave Interdisciplinary Application Division, Institute of Fluid Science, Tohoku University, \\ 2-1-1 Katahira, Aoba-ku, Sendai 980-8577, Miyagi, Japan
}

\begin{abstract}
This paper reports the result of a primary experimental and analytical study used to explore a reliable technology that is potentially applicable to the inactivation of micro-creatures contained in ship ballast water. A shock wave generated by the micro-explosion of a $10 \mathrm{mg}$ silver azide pellet in a $10 \mathrm{~mm}$ wide parallel test section was used to interact with a bubble cloud consisting of bubbles with average diameter $10 \mu \mathrm{m}$ produced by a swirling flow type micro-bubble generator. Observations were carried out with a high-speed camera, IMACON200, and the corresponding rebound pressures of the collapsing bubbles were measured with a fiber optic probe pressure transducer that provides high spatial and temporal resolutions. We found that micro-bubbles collapse in several hundred nanoseconds after the shock exposure and the resulting peak pressure pulses that repeatedly occurred exceeded well over $200 \mathrm{MPa}$ measured at the $20 \mathrm{~mm}$ distance from the explosion center. These continued for well over $20 \mu \mathrm{s}$. The experimental pressure responses were explained by solving the one-dimensional bubble Rayleigh-Plesset equation. Such high peak pressures could be used effectively for the inactivation of micro-creatures contained in ship ballast water.
\end{abstract}

Key words: Micro-bubble, Shock Wave, Flow Visualization, Compressible Flow, Gas-Liquid Two-Phase Flow, Rebound Pressure, Ship Ballast Water, Pressure Measurement

\section{Introduction}

To preserve the global marine ecological system ${ }^{(1)-(3)}$, the International Maritime Organization (IMO) adopted in 2004 a treaty governing the quantity of marine creatures contained in ship ballast water and called for observation of the regulation and its guidelines throughout the world ${ }^{(4)}$. Companies and institutes throughout the world then proposed and developed ballast water treatment systems that would satisfy the IMO regulation. Typical technologies are, for instance, known as photocatalytic sterilization, deoxidization sterilization with the presence of cavitation, ozone sterilization with cavitation, absorptive removal by using electromagnetic power, etc ${ }^{(5)}{ }^{(6)}$. Some of these have already been approved by the IMO. However, problems still remain regarding the cost of their installation and maintenance, and in addition, the use of chemical substances is not necessarily highly recommended.

Abe et al. ${ }^{(7)}{ }^{(8)}$ have proposed a method in which high pressures generated by impact have worked well for the establishment of a safe and harmless technology to sterilize

\footnotetext{
*Received 21 Jan., 2010 (No. T1-08-0871) Japanese Original : Trans. Jpn. Soc. Mech. Eng., Vol.75, No.752, B (2009), pp.668-675 (Received 26 Sep., 2008) [DOI: 10.1299/jfst.5.235]
}

Copyright $\odot 2010$ by JSME 
marine bacteria contained in ship ballast water and have used a gas gun for measuring shock resistivity of marine Vibrio sp. contained in a small culture solution. The result revealed that the bacteria were inactivated completely at shock over-pressures exceeding 200MPa. This shock sterilization was achieved not only from the mechanical effect induced by the high shock pressure exposure but also by the chemical effect associated with the formation of free radicals ${ }^{(9)-(11)}$. Then, the shock pressures generated by the collapsing bubbles were considered to be beneficial for effectively sterilizing a large volume of ship ballast water ${ }^{(12)}$. In particular, micro-bubbles remain in the water for a long duration and possibly generate free radicals when they contract ${ }^{(13)(14)}$. A project has therefore started to take advantage of micro-bubble collapse as an efficient means of treating ballast water. However, the dynamic response of micro-bubble clouds due to shock exposure in a confined space has not been well studied. Existing sterilizing methods using micro-bubbles are mostly based on either the toxicity of gases or deoxidization such as the use of ozone, carbon dioxide or nitrogen, while the mechanical effect of micro-bubble collapse has not been previously conceived.

The present paper reports the result of our recent experimental observation of micro-bubble exposure to external shock waves and pressure measurement during their collapse. It also considers the result of a one-dimensional analysis based on bubble dynamic modeling. The micro-bubble cloud at shock exposures eventually generated trains, sustained for over $20 \mu \mathrm{s}$, of high peak pressure pulses sufficient to inactivate marine bacteria. These are favorably applicable to practically to the treatment of ship ballast water.

\section{Experiment}

\subsection{Generation of Micro-bubbles}

We created micro-bubbles in a swirling flow type micro-bubble generator, M2-LM/SCS (Nanoplanet Inc.). This swirling flow created by the pump inflow decreased the pressure at the center core of the flow thereby swallowing air into the generator via a tube from the outside. The swirling air column was then fragmented into micro-bubbles due to the shear force at the flow exit. Its optimum operating condition was set empirically at $17 \mathrm{~L} / \mathrm{min}$ of delivery volume and $1 \mathrm{~L} / \mathrm{min}$ of air supply, respectively.

Figure 1 shows the arrangement of the present micro-bubble generation system and the micro-bubbles generated in $3 \mathrm{wt} \%$ salt water. The system was connected using a magnet pump MD-30RZ (Iwaki Co.), and air was supplied via a regulator. In the case of the $27 \mathrm{~L}$ salt water contained in a water tank, as shown in Fig. 1 (b), the test water became milky and bubbly after operating for about 30 s from its start. Water in the tank started to circulate, the bubbly water near the pump intake then being repeatedly sucked into the pump. Because of this, it is hard to accurately estimate the volume of air supplied into the micro-bubble generator.

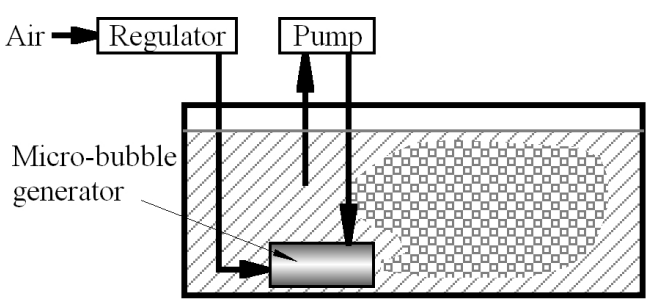

(a)

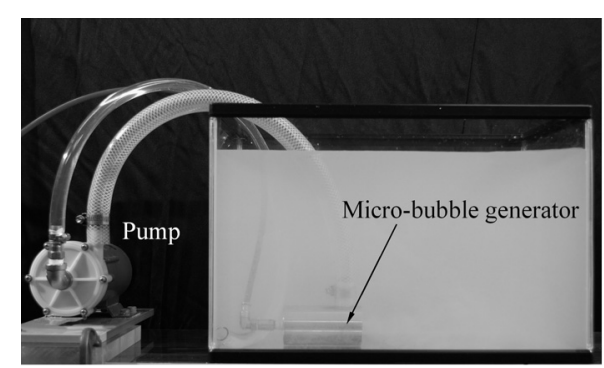

(b)

Fig. 1 Schematic diagram of the micro-bubble generation system and bubbly $3 \%$ salt water 


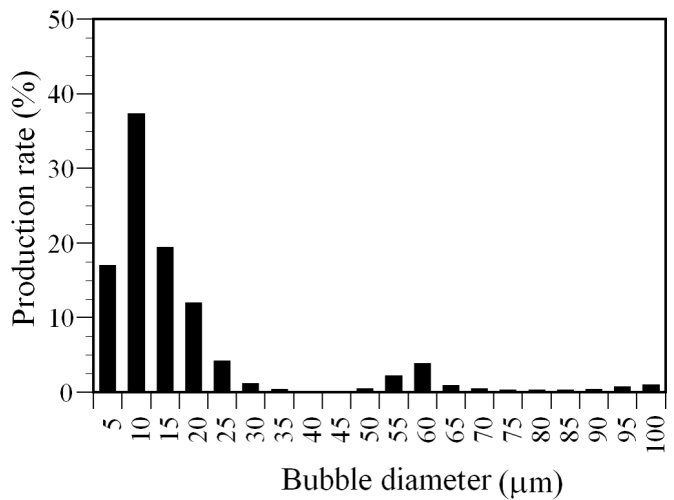

Fig. 2 Bubble size distributions measured by using a PDA system

The bubbles could not be uniformly dispersed in the tank water because the bubbly flow discharged from the generator was uncontrollable. Nevertheless, the bubbly water was sampled randomly at various positions in the tank and eventually the estimated average void ratio was found to be $1.1 \%$. This value was then used as a nominal void ratio.

Micro-bubble diameters were measured using a PDA system, (phase Doppler anemometry, Dantec dynamics), which derives bubble diameter by using Doppler and diffraction signals from the dispersion of an irradiating laser beam. The measured result showed that the diameters of $85 \%$ of the bubbles were smaller than $20 \mu \mathrm{m}$ and that the bubble diameters were distributed around $10 \mu \mathrm{m}$ as shown in Fig. 2. This result indicated that micro-bubbles were almost homogeneously distributed and equally sized.

\subsection{Observation of Micro-bubbles}

By trapping the bubbly water in a $300 \mu \mathrm{m}$ gap sandwiched between two glass plates, we observed the process of spontaneous contraction of the micro-bubbles. It took about $650 \mathrm{~s}$ for the bubbles to contract to their minimum detectable diameter of $3 \mu \mathrm{m}$, the bubbles being resolved in water and their rebound motion was never detected.

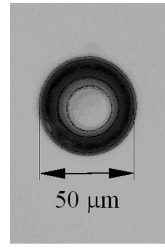

(a) $0 \mathrm{~s}$

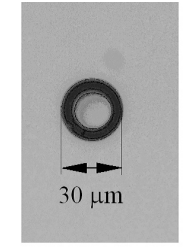

(b) $500 \mathrm{~s}$

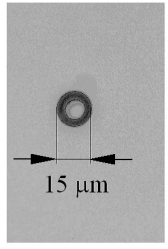

(c) $600 \mathrm{~s}$

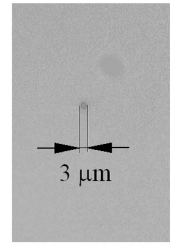

(d) $650 \mathrm{~s}$

Fig. 3 Natural contraction of a $50 \mu \mathrm{m}$ micro-bubble

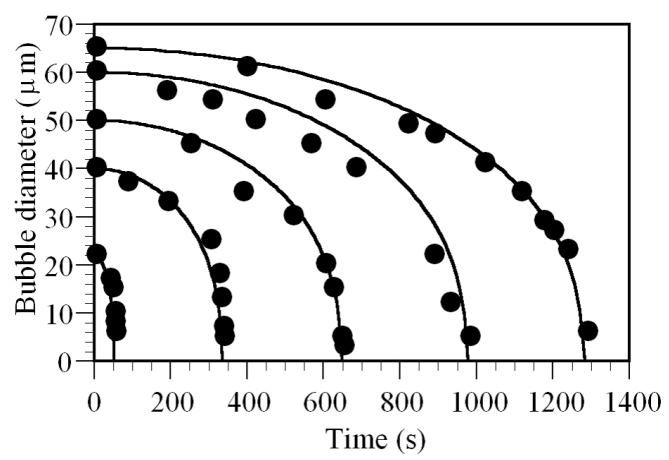

Fig. 4 Observation of micro-bubble diameter: filled circles designate measured data and solid lines the solutions of the Rayleigh-Plesset equation 
Figure 4 shows the temporal change of the bubble diameter for different initial bubble diameters. The ordinate designates the bubble diameter and the abscissa the elapsed time in $\mu \mathrm{s}$. The filled circles designate the measured data and the solid lines the analytical solutions obtained by solving the Rayleigh-Plesset equation, in which a single spherical bubble was assumed and the compressibility and viscosity of the water and the surface tension of bubbles were neglected ${ }^{(15)(16)}$. The solutions were derived for individual initial conditions of the theoretical pressure difference between the inside and outside of the bubble, these being estimated from the observed period of the bubble motion. In the case of a $65 \mu \mathrm{m}$ dia. bubble, it took about 1300 s to reach its minimum diameter. The negative gradient of the analytical curves implies an increase in the bubble contraction velocity. Analytical results agreed well with the experiments and no bubble rebound was observed. Hence, air and/or water vapor in the bubbles were resolved into the water while retaining thermal equilibrium. Takahashi et al. ${ }^{(13)(14)}$ have reported that such a slow contraction of micro-bubbles is one of the unique features of the bubble collapse phenomena. They confirmed, by electron spin-resonance (ESR) measurements using a spin-trapping reagent, that micro-bubbles can produce free radicals during their self-contraction when not exposed to any external pressures. However, the amount of free radicals so far generated by the self-contraction was not clarified. Previously we examined the inactivation of marine Vibrio sp. based on the self-contraction of micro-bubbles ${ }^{(12)}$, in which micro-bubbles were supplied continuously for 12 hours into $10 \mathrm{~L}$ seawater containing the marine bacteria, and found that the sterilization ratio was, at most, around $70 \%$. The result indicates that the self-contraction is not highly efficient for the inactivation of the marine bacteria. Hence, in order to gain higher efficiency of inactivation effect, both bubble motion and external pressure should be introduced.

\subsection{Experimental Setup}

In order to confirm the micro-bubble motion exposed to shock waves and high-pressure generation during micro-bubble collapse, we performed high-speed imaging and pressure measurements with higher spatial and temporal resolutions. A spherical shock wave was generated by a micro-explosion of a $10 \mathrm{mg}$ silver azide pellet, whose total energy was about 15J. The explosive was glued to a $0.6 \mathrm{~mm}$ diameter optical fiber tip and ignited through it by irradiation from a Q-switch Nd:YAG laser beam of $20 \mathrm{~mJ} /$ pulse and $7 \mathrm{~ns}$ pulse duration. About one third of the total energy of the explosion was successfully converted to generating the shock wave ${ }^{(17)}$ and hence the extra addition of laser energy contributed insignificantly to the shock wave formation.

The experimental setup and optical arrangement are shown in Fig. 5. As a wide test section would obscure images of the individual micro-bubbles during their motion, we adopted a narrower test chamber consisting of two $8 \mathrm{~mm}$ thick acrylic plates separated by a $10 \mathrm{~mm}$ interval. Its view field was $110 \mathrm{~mm} \times 110 \mathrm{~mm}$. The micro-explosive was placed at its center. An initial spherical shock wave immediately became cylindrical as it was reflected many times from the two walls separated within such a small space. Micro-bubbles already produced in another water tank were supplied by circulation into the test chamber, this being suspended for 10 to 20 s so as to calm down the bubble motion. An image converter camera IMACON200 (DRS Hadland Co.) recorded the visualized images, which warrants 14 images maximum at its shortest exposure time of 5ns. A fiber optic probe hydrophone, FOPH2000 (RP Acoustics Co.) consisting of a $100 \mu \mathrm{m}$ core diameter optical fiber and with a response frequency of $100 \mathrm{MHz}$ recorded shock over-pressures. The optical fiber was inserted $20 \mathrm{~mm}$ from the micro-explosive as shown in Fig. 6. 


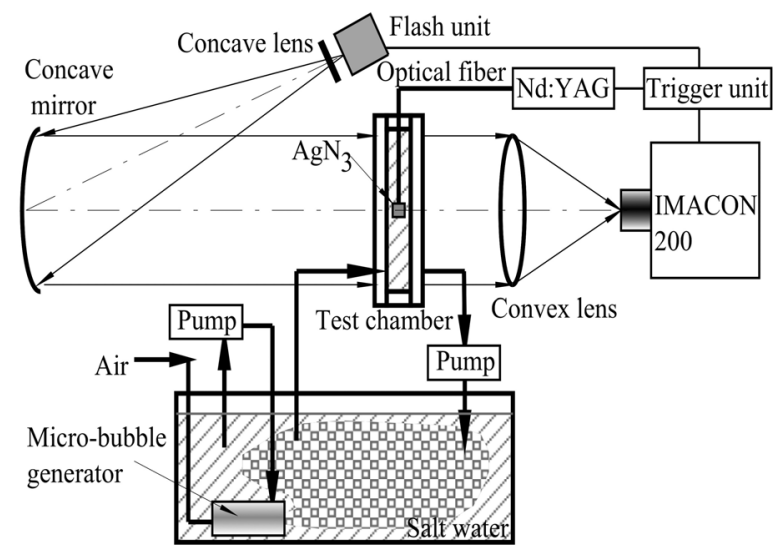

Fig. 5 Experimental setup

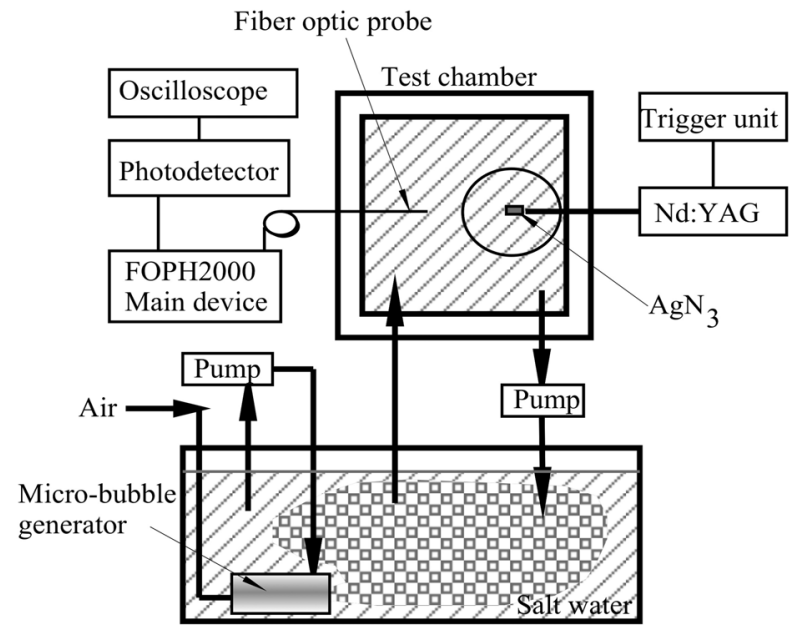

Fig. 6 Pressure measurement by using FOPH2000

\section{Results and Discussion}

\subsection{Bubble Motion Analysis}

By using a double exposure holographic interferometer, the interaction between an underwater spherical shock wave and a $1.7 \mathrm{~mm}$ dia. air bubble was observed for the first time and the temporal variations of the pressure distributions around the bubble were estimated by analyzing the fringe patterns ${ }^{(18)}$. The results revealed that at the shock impingement on a bubble, an expansion wave was reflected and simultaneously the bubble contraction started with an increasing the pressure at the spot on the bubble surface at which the shock at first impinged. High pressures, locally enhanced, coalesced into a shock wave and drove a micro-water jet that penetrated the contacting bubble. The bubble quickly recovered from its minimum volume but the micro-water jet continued its penetration.

As for the shock/micro-bubble interaction, the behavior of the present micro-bubbles seems to be in principle identical to that of $1.7 \mathrm{~mm}$ diameter bubbles. However, the shock interaction with the present micro-bubbles has a different time scale from that of larger bubbles because the surface tension would be comparatively large in the case of micro-bubbles and hence would cause more complex bubble interaction. We decided to analyze a single spherical micro-bubble for the sake of simplicity in solving the one-dimensional equation of bubble motion. We then estimated approximately the condition of the micro-bubble collapse and the pressure variations around the micro-bubble during 
motion.

The equation for a spherical bubble in quiescent compressible fluid was solved neglecting thermal and diffusion effects ${ }^{(19)(20)}$. The time variations of the bubble diameter and its pressure at different shock exposure pressures were estimated. Figures 7 (a) and (b) show the time variations of bubble radii and bubble pressures initiated by $1 \mathrm{MPa}$ to $5 \mathrm{MPa}$ pressure loading on $50 \mu \mathrm{m}$ dia. bubbles, respectively. Although the model is too robust to

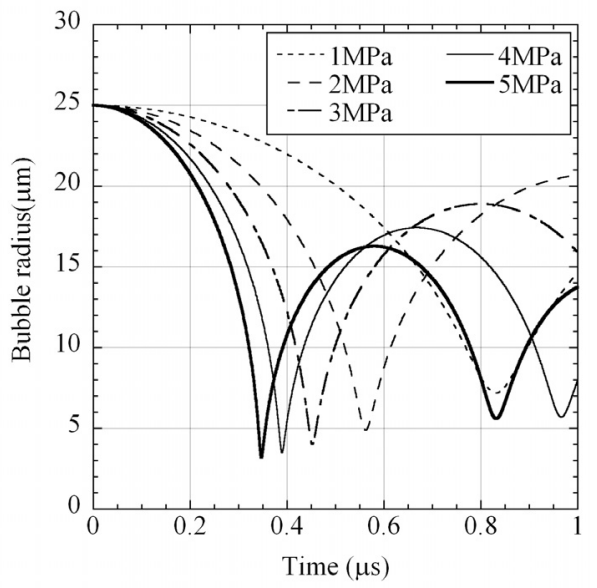

(a)

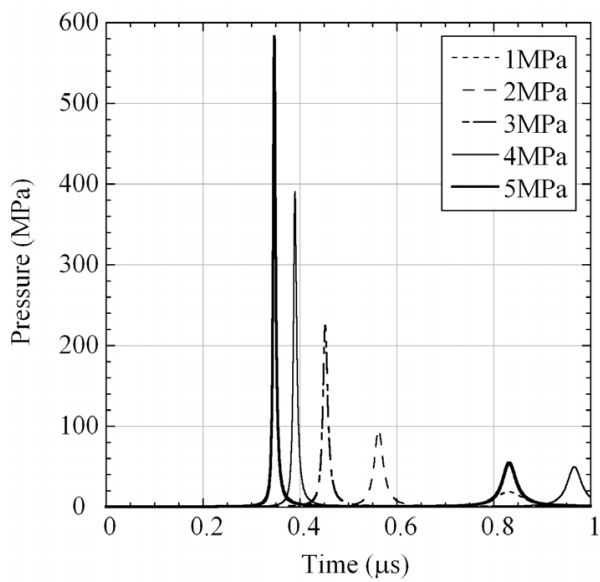

(b)

Fig. 7 Analytical solutions of the bubble dynamic equations at different conditions of ambient pressure

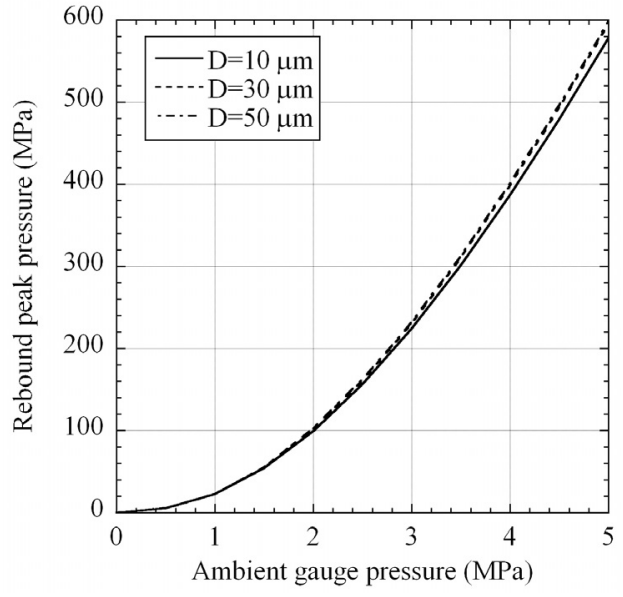

Fig. 8 Relation between rebound peak pressure and ambient gauge pressure 
reproduce the real motion of the micro-bubbles upon shock impingement, nevertheless it is still useful to predict the entire course of the micro-bubble motion. In Fig. 7 (a), the first micro-bubble rebound occurs within $1 \mu$ s and the collapsing interval becomes shorter with increasing ambient pressure when the pressure is higher than $1 \mathrm{MPa}$. In addition, the bubble pressures are a maximum at the first rebound. The rebound pressure becomes high with an increase in the loading pressure, and its value well exceeds several hundred MPa when the loading pressure is higher than $2 \mathrm{MPa}$.

Figure 8 shows the relationship between peak pressures obtained at the first rebound and the ambient pressure for 10,30 and 50 $\mu \mathrm{m}$ dia. micro-bubbles. The ordinate designates the rebound pressure in MPa and the abscissa the loading pressure in MPa. Results so far obtained do not depend significantly on the bubble diameter under study. The result implies that we should load to at least $3 \mathrm{MPa}$ to achieve the maximum rebound pressure of $200 \mathrm{MPa}$ needed, which is known by our previous study to inactivate marine bacteria.

\subsection{Interaction between Micro-bubbles and Cylindrical Shock Wave}

Figure 9 shows sequential images of a cylindrical shock wave interaction with micro-bubbles. The inter-frame time was $2 \mu \mathrm{s}$, the exposure time was $50 \mathrm{~ns}$, and the frame width was $56 \mathrm{~mm}$. These pictures are slightly underexposed due to the distributed micro-bubbles, and the minimum micro-bubble diameter that we could distinguish directly from these pictures was 600 to $800 \mu \mathrm{m}$. In Fig. 9, we can see the explosion product gas, cylindrical shock wave CS and an elastic stress wave ES propagating in the acrylic window material. CS propagates at about $1500 \mathrm{~m} / \mathrm{s}$ and ES about $2100 \mathrm{~m} / \mathrm{s}$. A $700 \mu \mathrm{m}$ dia. bubble B visible at about $12 \mathrm{~mm}$ on the right from the ignition position started to contract immediately after the shock impingement. A rebound cylindrical shock wave RS generated by the bubble B was not clear but is observable in Figs. 9 (f) and (g), while the rebound phenomena of micro-bubbles less than $100 \mu \mathrm{m}$ in diameter was not identified. It should be noticed that multiple reflected shock waves from the acrylic side plates also propagate behind CS, though almost invisible or very vaguely identified in the images in Fig. 9.
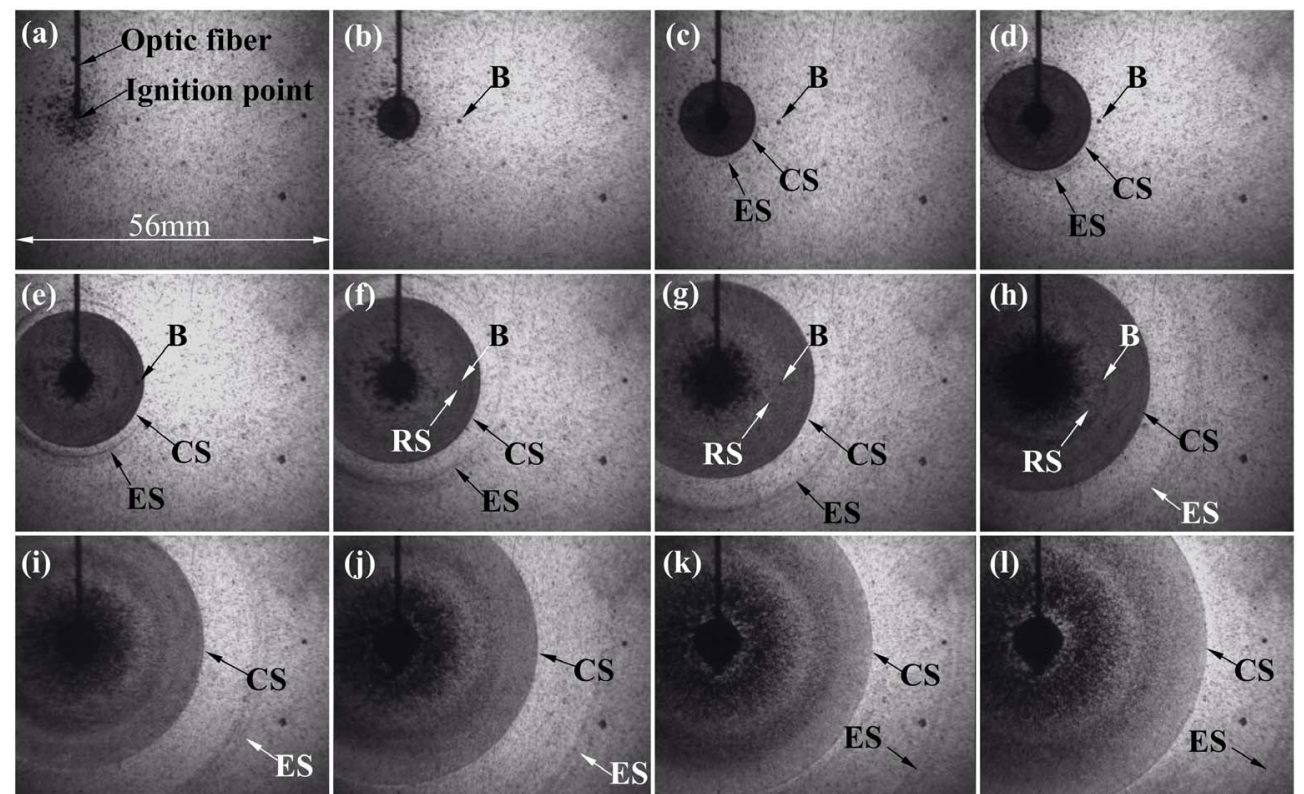

Fig. 9 Interaction between micro-bubbles and a cylindrical shock wave generated by micro-explosion of $10 \mathrm{mg} \mathrm{AgN}_{3}$ in the bubbly test chamber: a $700 \mu \mathrm{m}$ dia. bubble B, a cylindrical shock wave CS, an elastic stress wave in the acrylic windows ES, and a rebound shock wave RS 


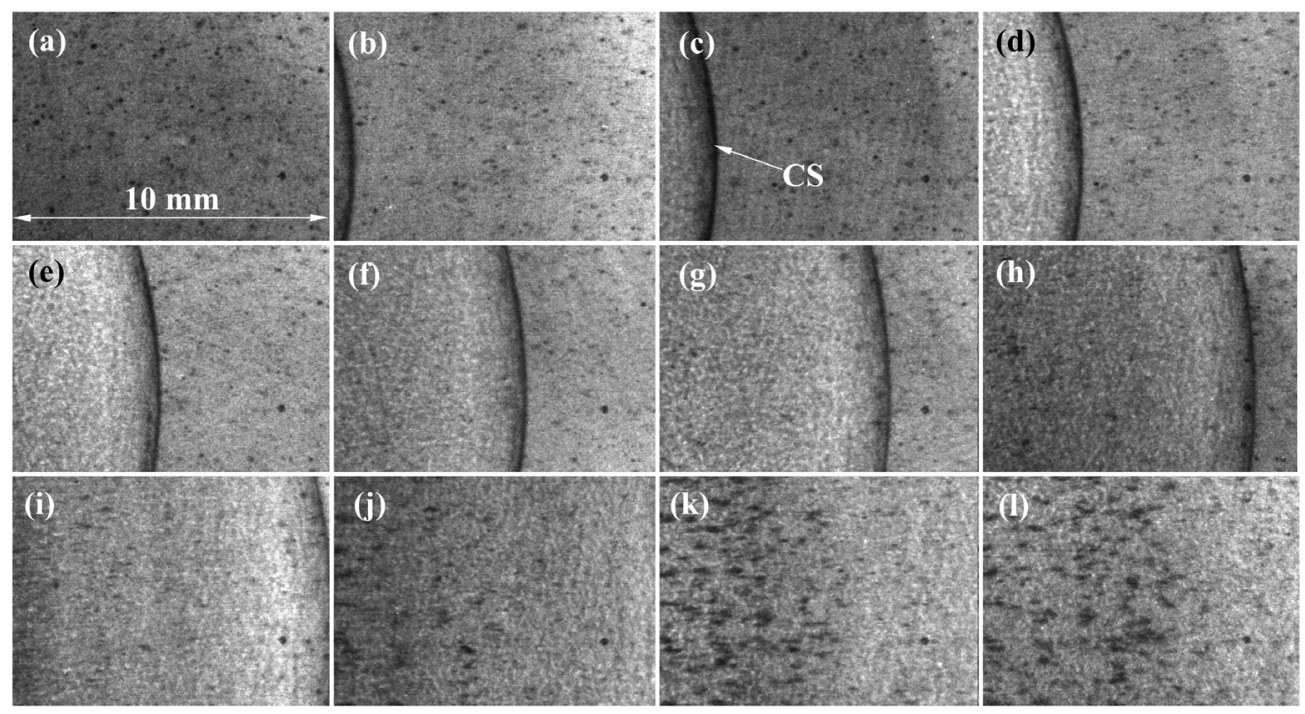

Fig. 10 Close-up observation of shock wave interaction with micro-bubbles

Figure 10 shows the sequential magnified images at a $1 \mu$ s frame interval, 50ns exposure time and view field of $10 \mathrm{~mm}$. CS propagates left to right and micro-bubbles in front of CS are shown as dark dots. In Figs. 10 (d) and (e), the dark dots disappeared immediately behind CS and were obscured. Such a sudden contrasting change was due to the local change in the refractive index of the bubbly water, that is, to the density enhancement associated with the micro-bubble collapse. After impingement of CS, the region of collapsing bubbles starts to expand, so that the shadows of micro-bubbles were gradually enlarged as seen in Figs. 10 (f) to (l). This observation confirms that the micro-bubbles started to recover within $1 \mu$ s after CS passage.

\subsection{Measurement of Rebound Pressure}

Figure 11 presents a pressure history measured by FOPH2000. Figures 11 (a) and (b) are cases in water without micro-bubbles and with bubbly water, respectively. The pressure increases at CS and its maximum value reaches about $50 \mathrm{MPa}$ as shown in Fig. 11 (a). This value is even higher than that of a spherical shock because the spherical shock in water in an infinitely large chamber readily attenuates, whereas CS being two-dimensional is less affected by geometrical attenuation. A precursory pressure increase of about $5 \mathrm{MPa}$ was observed at about $200 \mathrm{~ns}$ before the arrival of CS. As mentioned above, this is caused by the release of the precursory elastic stress wave propagating in the acrylic plates.

In the case of bubbly water as shown in Fig. 11 (b), precursory pressures of 20 to $30 \mathrm{MPa}$ are measured in front of CS, which apparently represent the release of the stress waves. At about $1 \mu$ s after CS passing, a jump of CS over-pressures of about 60 and $100 \mathrm{MPa}$ follows. Then peak over-pressures substantially exceeding 200MPa appear, these fluctuating around $150 \mathrm{MPa}$ and are maintained for several tens of $\mu$ s. It should be noticed again that micro-bubble collapse indeed created these higher over-pressure trains shown in Fig. 11 (a), which have not been observed before.

We presume that the high pressures randomly generated at bubble collapse would be repeatedly reflected between the narrow test sections and result in the peak pressure train. Hence, a frequency analysis was applied to the pressure history. The bubble collapse was caused not only by CS loading but also by the repetition of the reflected wave created during the transition from the spherical shock wave to CS. Figure 11 (c) shows the result of a frequency analysis using the Fast Fourier Transformation of the pressure history. The 
ordinate designates the spectrum and the abscissa the frequency in $\mathrm{kHz}$. The peak spectrum was at $164 \mathrm{kHz}$. As the propagation speed of the underwater shock wave is about $1500 \mathrm{~m} / \mathrm{s}$, the frequency of its reflection over the $10 \mathrm{~mm}$ wide test section corresponds to about $150 \mathrm{kHz}$. This value was found to be very close to the frequency at the peak spectrum in Fig. 11 (c).

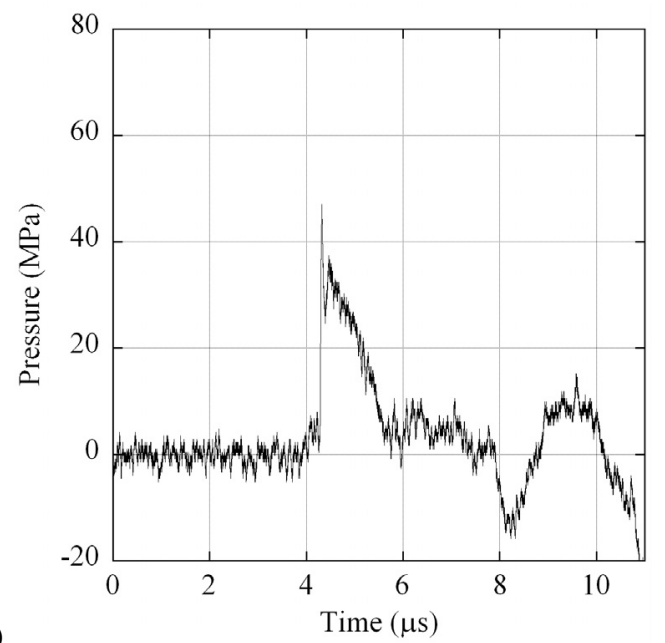

(a)

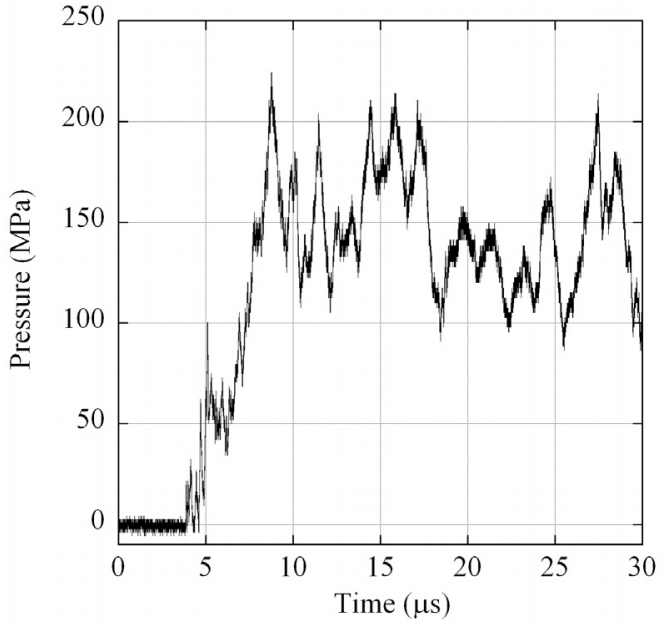

(b)

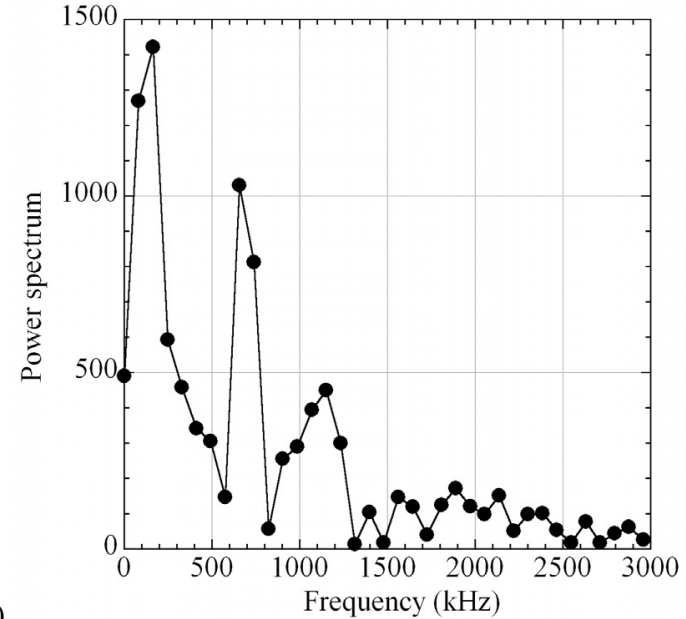

(c)

Fig. 11 Measured pressure with FOPH2000 in the test chamber, (a) without micro-bubbles, (b) with micro-bubbles, and (c) FFT diagram of (b) 
Due to such a successive shock wave loading in the narrow test chamber, micro-bubbles repeatedly start to contract and expand, and these contribute eventually to high peak pressures maintained for a long duration of time. This finding would suggest that for a sterilization apparatus design, the use of micro-bubble collapse under shock wave loading in a confined space could generate and maintain a high enough pressure to inactivate marine bacteria.

Figure 12 shows analytical results on radius and pressure variation of single spherical bubbles of 10 and $30 \mu \mathrm{m}$ initial diameter exposed to the over-pressure represented in Fig. 11 (a). The first rebound occurs at $110 \mathrm{~ns}$ for the $10 \mu \mathrm{m}$ dia. bubble and $260 \mathrm{~ns}$ for the $30 \mu \mathrm{m}$ dia. bubble, respectively. The maximum rebound pressures are around $400 \mathrm{MPa}$ in both cases. These analytical results agreed approximately with the present observation that the first rebound of a micro-bubble took place in sub $\mu$ s time after the shock wave loading and its peak over-pressure exceeded well over several hundred MPa.

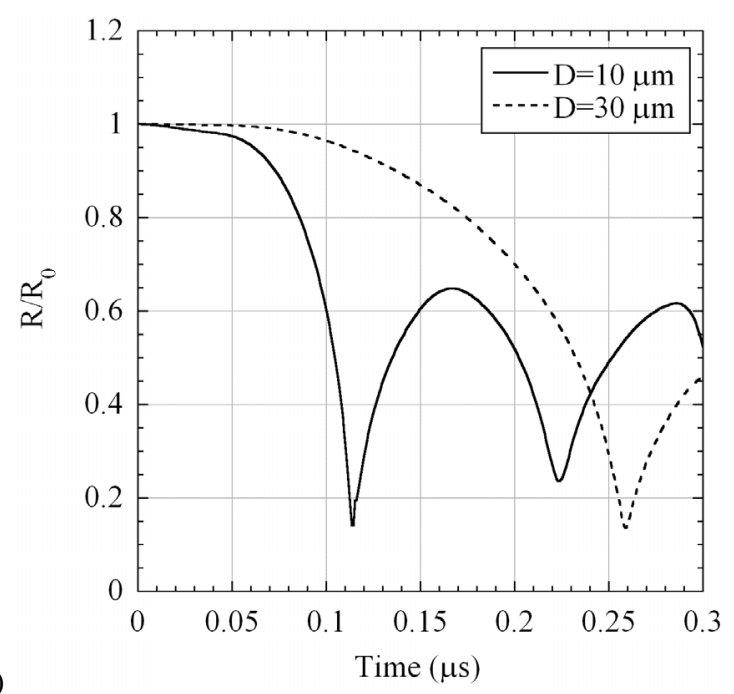

(a)

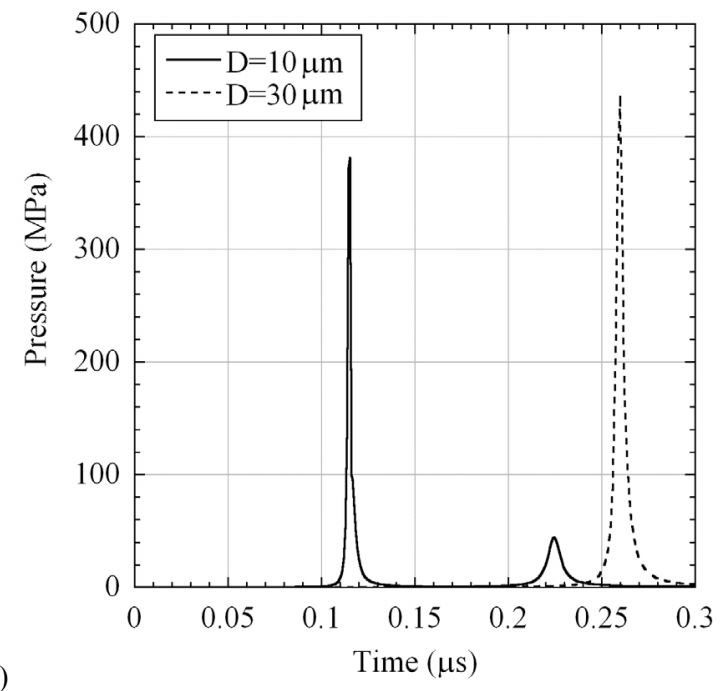

Fig. 12 Analytical solution of bubble motion calculated by using pressure data measured with FOPH2000 


\section{Conclusions}

By performing high speed recording of shadowgraph images of shock wave/ micro-bubble interactions in a confined space and pressure measurements with high spatial and temporal resolutions, we found that shock wave induced collapse of micro-bubbles repeatedly produced a train of peak pressures exceeding well over 200MPa. A repetition of pressure spikes continued for a long time duration which would effectively inactivate marine bacteria. This high-pressure generation would be useful for developing a sterilization technology to efficiently treat a large volume of ballast water. For establishing such a technology, we have to optimize the void ratio and test section geometry and to determine the method of obtaining underwater shock wave generation.

\section{Acknowledgements}

The authors wish to acknowledge that this project was supported by the advanced research grants for the research project entitled "the creation of an international marine transportation system integrating three-principles of transportation" offered by the Ministry of Education, Culture, Sports, Science and Technology of Japan.

\section{References}

(1) Fukuyo, Y., Harmful Transfer of Organisms by Ballast Water, Science of Ship and the Sea, Vol. 7, (2004), pp. 30-33.

(2) Kikuchi, T., What is the Issue of Ship's Ballast Water?, Sea and Safety (in Japanese), No.67, (2001), pp. 2-9.

(3) Ruiz, G. M., Rawlings, T. K., Dobbs, F. C., Drake, L. A., Mullady, T., Huq, A., Colwell, R. R., Global Spread of Microorganisms by Ships, Nature, Vol. 408, (2000), pp. 49-50.

(4) International Maritime Organization. "International Convention for the Control and Management of Ships' Ballast Water and Sediments adopted in 2004". Ballast water management. (online), available from <http://www.imo.org/home.asp>, (accessed 2008-4-1).

(5) Kikuchi, T., Yoshida, K., Kino, S., Fukuyo, Y., Progress Report on the 'Special Pipe System' as a Potential Mechanical Treatment for Ballast Water, 2nd International Ballast Water Treatment R\&D Symposium, IMO London, (2003), pp. 19-25.

(6) Saho, N., Isogami, H., Mizumori, T., Nishijima, N., Superconduction magnetic separator for ballast-water treatment, 2nd International Ballast Water Treatment R\&D Symposium, IMO London, (2003), pp. 125-134.

(7) Abe, A., Mimura, H., Ikeda, T., Katakura, R., Shock Compression of Marine Bacterial Cells Enclosed in Aluminum Container, Journal of the JIME, Vol.40, No. 2, (2005), pp. 110-115.

(8) Abe, A., Kawasaki, H., Study on Shock Wave Propagation under Saltwater Sealed in Aluminum Containers, Transactions of the Japan Society of Mechanical Engineers, Series $B$, Vol.72, No.722, (2006), pp. 2418-2424.

(9) Abe, A., Mimura, H., Ishida, H., Yoshida, K., The effect of shock pressures on the inactivation of a marine Vibrio sp., Shock Waves, Vol.17, No.1-2, (2007), pp. 143-151.

(10) Mimura, H., Abe, A., Yoshida, K., Ishida, H., Changes in the Number of Colony-Forming Marine Vibrio sp. Cells After Exposure to Shock Pressures in the Presence of Sodium Ascorbate, Bull. Soc. Sea Water Sci., Jpn, Vol.61, No.2, (2007), pp. 132-133.

(11) Mimura, H., Abe, A., Ishida, H., Changes of marine bacterial populations in a ship's ballast water and sediment, and application of shock pressures to complete killing of Vibrio sp. cells isolated from ballast water, Environmental Microbiology Research Trends edited by G. V. Kurladze, (2007), pp. 191-212. 
(12) Abe, A., Kanai, H., Mimura, H., Nishio, S., Ishida, H., Study on application of shock waves generated by micro bubbles to the treatment of ships' ballast water, Proc. 26th International Symposium on Shock Waves, (2007), A4890.

(13) Takahashi, M., Chiba, K., Li, P., Free-Radical Generation from Collapsing Microbubbles in the Absence of a Dynamic Stimulus, J. Phys. Chem. B, Vol.111, No.6, (2007), pp. 1343-1347.

(14) Takahashi, M., Chiba, K., Li, P., Formation of Hydroxyl Radicals by Collapsing Ozone Microbubbles under Strongly Acidic Conditions, J. Phys. Chem. B, Vol.111, No.39, (2007), pp. 11443-11446.

(15) Rayleigh, Lord., On the pressure developed in a liquid during the collapse of a spherical cavity, Phil. Mag., Vol.34, (1917), pp. 94-98.

(16) Plesset, M. S., The Dynamics of Cavitation Bubbles, J. Appl. Mech., Vol.16, (1949), pp. 277-282.

(17) Takayama, K., Holographic interferometric study of shock wave propagation in two-phase media, Proc. 16th Int. Symp. Shock Tubes Waves, (1987), pp. 51-62.

(18) Abe, A., Shock wave refraction and flow from an openended shock tube (in Japanese), Doctoral thesis, Graduate School of Tohoku University, (1991), pp. 42-44.

(19) Tomita, Y., Shima, A., Mechanisms of impulsive pressure generation and damage pit formation by bubble collapse, J. Fluid Mech., Vol.169, (1986), pp. 535-564.

(20) Shima, A., Studies on bubble dynamics, Shock Waves, Vol.7, (1997), pp. 33-42. 